\title{
Risk Factors for Mobility Decline in Community-Dwelling Older Adults: A Systematic Literature Review
}

\author{
Philippa J.A. Nicolson, Maria T. Sanchez-Santos, Julie Bruce, Shona Kirtley, \\ Lesley Ward, Esther Williamson, and Sarah E. Lamb
}

\begin{abstract}
Mobility is essential to maintaining independence for older adults. This systematic review aimed to summarize evidence about self-reported risk factors for self-reported mobility decline; and to provide an overview of published prognostic models for selfreported mobility decline among community-dwelling older adults. Databases were searched from inception to June 2 , 2020. Studies were screened by two independent reviewers who extracted data and assessed study quality. Sixty-one studies (45,187 participants) were included, providing information on 107 risk factors. High-quality evidence and moderate/large effect sizes for the association with mobility decline were found for older age beyond 75 years, the presence of widespread pain, and mobility modifications. Moderate-high quality evidence and small effect sizes were found for a further 21 factors. Three model development studies demonstrated acceptable model performance, limited by high risk of bias. These findings should be considered in intervention development, and in developing a prediction instrument for practical application.
\end{abstract}

Keywords: mobility disability, predictors, self-reported

Mobility is defined as "the ability to move oneself within community environments that expand from one's home, to the neighborhood, and to regions beyond" (Webber, Porter, \& Menec, 2010). Maintaining mobility is fundamental to aging well for older adults (Guralnik et al., 1993). Limited mobility is linked to functional decline, mortality, and increased health care costs (Guralnik et al., 1993). Changes in mobility are important as conceptually they precede disability within models of disablement and are potential targets for rehabilitation (Verbrugge \& Jette, 1994).

Mobility may be assessed using objective or self-reported measures. While it is acknowledged that different aspects of mobility may be measured with each, multiple studies have found a moderate correlation between the two approaches (Alexander et al., 2000; Syddall, Westbury, Cooper, \& Sayer, 2015). Selfreported mobility is important as it is the most commonly used measure clinically (Chung, Demiris, \& Thompson, 2015). Objective physical performance measures do not reflect the individual's perceptions about their own mobility, and are most often conducted in a supervised, controlled environment, thus unreflective of a real-

(C) 2021 The Authors. Published by Human Kinetics, Inc. This is an Open Access article distributed under the terms of the Creative Commons Attribution 4.0 International License, CC BY 4.0, which permits unrestricted noncommercial and commercial use, distribution, and reproduction in any medium, provided the original work is properly cited, the new use includes a link to the license, and any changes are indicated. See http://creativecommons.org/licenses/by/4.0. This license does not cover any third-party material that may appear with permission in the article.

Nicolson, Sanchez-Santos, Kirtley, Williamson, and Lamb are with the Nuffield Department of Orthopaedics, Rheumatology and Musculoskeletal Sciences (NDORMS), University of Oxford, Oxford, United Kingdom. Bruce is with the Warwick Clinical Trials Unit, Warwick Medical School, University of Warwick, Coventry, United Kingdom. Ward is with the Department of Sport, Exercise \& Rehabilitation, Northumbria University, Newcastle upon Tyne, United Kingdom. Lamb is also with the College of Medicine and Health, University of Exeter, Exeter, United Kingdom. Nicolson (philippa.nicolson@ndorms.ox.ac.uk) is corresponding author. world situation. Potential risk factors for mobility decline may also be measured objectively or by self-report. Self-reported measures of these factors are also important, as they have low response burden, can capture both current and historical information, and can assess psychological and social factors.

Two previous reviews of risk factors for mobility decline combined objective and self-reported measures of risk factors and mobility, but these were narrative rather than systematic reviews (Rantakokko, Mänty, \& Rantanen, 2013; Yeom, Fleury, \& Keller, 2008). Consolidated evidence of self-reported risk factors contributing to self-reported mobility decline is needed to inform clinical decision making and to inform the development of a prognostic tool to help clinicians and researchers identify older adults at risk of mobility decline. The primary objective of this systematic review was to synthesize available evidence for self-reported factors which predict decline in self-reported mobility after 12 months to 5 years of follow-up among community-dwelling older adults. The secondary objective was to identify, describe, and synthesize the predictive accuracy of prognostic models developed to predict risk of selfreported mobility decline in community-dwelling older adults.

\section{Methods}

This systematic review is registered at the International Prospective Register of Systematic Reviews database (PROSPERO 2019 CRD42019135420. Available from: https://www.crd.york.ac.uk/ prospero/display_record.php?ID=CRD42019135420). The review is reported according to the Preferred Reporting Items for Systematic Review and Meta-Analyses guidelines (Moher, Liberati, Tetzlaff, \& Altman, 2009), and the CHecklist for critical Appraisal and data extraction for systematic Reviews of prediction Modelling Studies (CHARMS) (Moons et al., 2014).

\section{Search Strategy}

The search strategy was developed conjointly with an information scientist (SK). We searched the MEDLINE (via OVID), EMBASE 
(via OVID), PsycINFO (via OVID), and CINAHL (via EBSCOhost) databases to identify studies that assessed the predictive value of selfreported risk factors on self-reported mobility decline. Search terms included controlled vocabulary (e.g., MeSH) terms and free-text terms. No date or language limits were applied to the search. The search strategy for MEDLINE is presented in Supplementary Table S1 (available online), and this was translated to the relevant controlled vocabulary headings and syntax for each database. The search was conducted on June 26, 2019, with an update run on June 2, 2020. Supplementary searches of the reference and citation lists of included studies and relevant systematic reviews were undertaken to identify additional eligible studies.

\section{Study Selection}

\section{Inclusion criteria.}

- Studies involving community-dwelling older adults with a minimum mean age of 60 years at recruitment.

- Longitudinal cohort studies evaluating the ability of self-reported factors to predict self-reported mobility decline. Randomized controlled trials were eligible if they provided appropriate data and treatment-effect modification analyses were reported.

- Prognostic model studies, with or without validation. Models had to contain at least two self-reported risk factors, and include age and sex as a minimum of adjustment factors.

- A self-reported measure of mobility, defined as difficulty with walking and/or difficulty in climbing stairs and/or life-space assessment, assessed at baseline and follow-up.

- A clear definition of mobility decline.

- Where a study collected both self-reported measures and objective measures, the study was eligible if data reported the relationship between self-reported measures and selfreported outcomes.

- Follow-up period of between 12 months and 5 years.

- Published in English, Spanish, or Portuguese (languages spoken by the research team).

\section{Exclusion criteria.}

- Studies including only patients with a specific medical condition (e.g., Parkinson's disease, dementia, stroke).

- Studies conducted in residential aged care settings.

- Studies published in abstract form only.

Search results were imported into Covidence (Veritas Health Innovation, Melbourne, Australia, www.covidence.org) for screening. Titles and abstracts of all identified citations were independently screened by two reviewers (PN and MS). Following title and abstract screening, the full text of all potentially eligible articles was retrieved and screened independently by the same two reviewers. Inclusion disagreements were resolved through discussion, with an adjudicator (PN and MS) available.

\section{Data Extraction}

A data extraction form in Microsoft Excel (Microsoft Corporation, Redmond, WA) was developed and piloted independently by two reviewers before independent data extraction from all articles (PN and MS). Disagreements were resolved by discussion.

Studies were categorized as etiological, predictor finding, or model development using the following definitions (Moons, Royston, Vergouwe, Grobbee, \& Altman, 2009):
- Etiological studies: A study examining causal association between predefined risk factor/s and mobility decline.

- Predictor finding studies: A study examining the contribution of multiple risk factors to predict mobility decline, without quantification of the predictive ability of the model.

- Model development studies: A study that aimed to develop a multivariable prediction model, assigning weighting to each identified risk factor and developing a final prediction model. These studies may or may not include internal validation studies.

Data extracted from all studies included: country of origin, year of study conduct, recruited and analyzed sample sizes, study design, type of study, length of follow-up, and participant characteristics (population source and setting, eligibility criteria, recruitment method, and sociodemographic characteristics). Method of assessment was recorded for each risk factor and mobility outcome.

For etiological studies, we extracted data for the specific risk factor/s of interest as stated in the study. For predictor finding studies, we extracted data on all included risk factors. We extracted unadjusted and adjusted effect estimates (odds ratio [OR], relative risk [RR], or hazard ratio [HR]) and corresponding $S E$ or confidence intervals for each risk factor. For model development studies, we extracted modeling method, handling of risk factors and missing data, method for selection of risk factors, model performance measures, and model evaluation. We contacted authors when insufficient information was reported.

\section{Assessment of Risk of Bias in Included Studies}

Two reviewers (PN and MS) independently assessed the risk of bias in included studies using the Quality In Prognosis Studies tool for etiological and predictor finding studies and the Prediction model Risk of Bias Assessment Tool for model development studies (Hayden, van der Windt, Cartwright, Cote, \& Bombardier, 2013; Wolff et al., 2019).

The Quality In Prognosis Studies tool assesses risk of bias across six domains: study participation, study attrition, risk factor measurement, outcome measurement, study confounding, and statistical analysis and reporting (Hayden et al., 2013). Each domain was rated as low, moderate, or high risk of bias (Hayden et al., 2013). The Prediction model Risk of Bias Assessment tool includes 20 signaling questions grouped within four domains (participant selection, predictors, outcome, and analysis) to evaluate the risk of bias of prediction models (Wolff et al., 2019). Studies were classified as low risk of bias if all domains were rated as low risk of bias. Any disagreements in scoring were resolved through discussion.

\section{Data Synthesis and Analysis}

Descriptive characteristics of all included studies were summarized and assessed for suitability for meta-analysis by considering the clinical heterogeneity of included studies based on population, definition of risk factors and/or mobility decline, and methodology (Riley et al., 2019). Adjusted effect estimates (minimum adjustment for age and gender/sex) were reported in the narrative analysis. Odds ratios of 1.4-2.5 were considered as small effect, 2.5-4.25 were classified as moderate, and 4.25 or greater as large (Chinn, 2000). Odds ratios were considered to indicate no association if the $95 \%$ confidence interval crossed 1.0. Information from risk factor and prognostic model studies were evaluated separately because their objectives and model-building methods are different (van den Berg et al., 2013). Performance of prognostic models were summarized and presented narratively. 


\section{Narrative Analysis}

We applied the following criteria proposed by Hayden, Tougas, Riley, Iles, and Pincus (2014) to categorize the consistency of evidence for each risk factor. Consistent evidence defined as two or more studies from two or more cohorts with $\geq 75 \%$ of studies showing the same direction of effect (i.e., four studies from three cohorts, of which three studies show the same direction of effect). Limited evidence defined as: single study or multiple studies from the same cohort. Inconsistent evidence defined as: two or more studies from two or more cohorts with $<75 \%$ of studies showing the same direction of effect (i.e., four studies from three cohorts, of which two studies show the same direction of effect).

\section{Assessment of Quality of Evidence}

The quality of the body of evidence was synthesized using the Grading of Recommendations Assessment, Development and Evaluation approach modified for use in reviews of risk factor studies (Huguet et al., 2013). Overall quality of evidence was rated as high, moderate, low, or very low considering study limitations, inconsistency of results, indirectness, imprecision, publication bias, moderate/large effect size, and the presence of a dose effect.

\section{Results}

\section{Study Selection}

The process of study selection is shown in Figure 1. In total, 26,079 citations $(18,201$ with duplicates removed) were identified. Of these, 187 articles progressed to full-text review, and 61 studies were included in the review. Among included studies, 47 were classified as etiological studies, 11 as predictor finding studies, and three were model development studies.

\section{Characteristics of Included Studies}

Characteristics and details of the 61 included studies are presented in Supplementary Table S2 (available online). Studies were published between 1993 and 2019. A total of 45,187 participants were analyzed. Included studies were all secondary analyses of data derived from 25 established cohorts. The cohorts from which the highest number of studies originated were the Nordic Research on Ageing Study (11 studies; Avlund, Damsgaard, \& Osler, 2004; Avlund, Damsgaard, Sakari-Rantala, Laukkanen, \& Schroll, 2002; Avlund, Davidsen, \& Schultz-Larsen, 1995; Avlund, Lund, Holstein, \& Due, 2004; Avlund, Osler, Damsgaard, Christensen, \& Schroll, 2000; Avlund, Pedersen, \& Schroll, 2003; Avlund, Vass, \& Hendriksen, 2003; Jørgensen, Lund, Siersma, \& Nilsson,

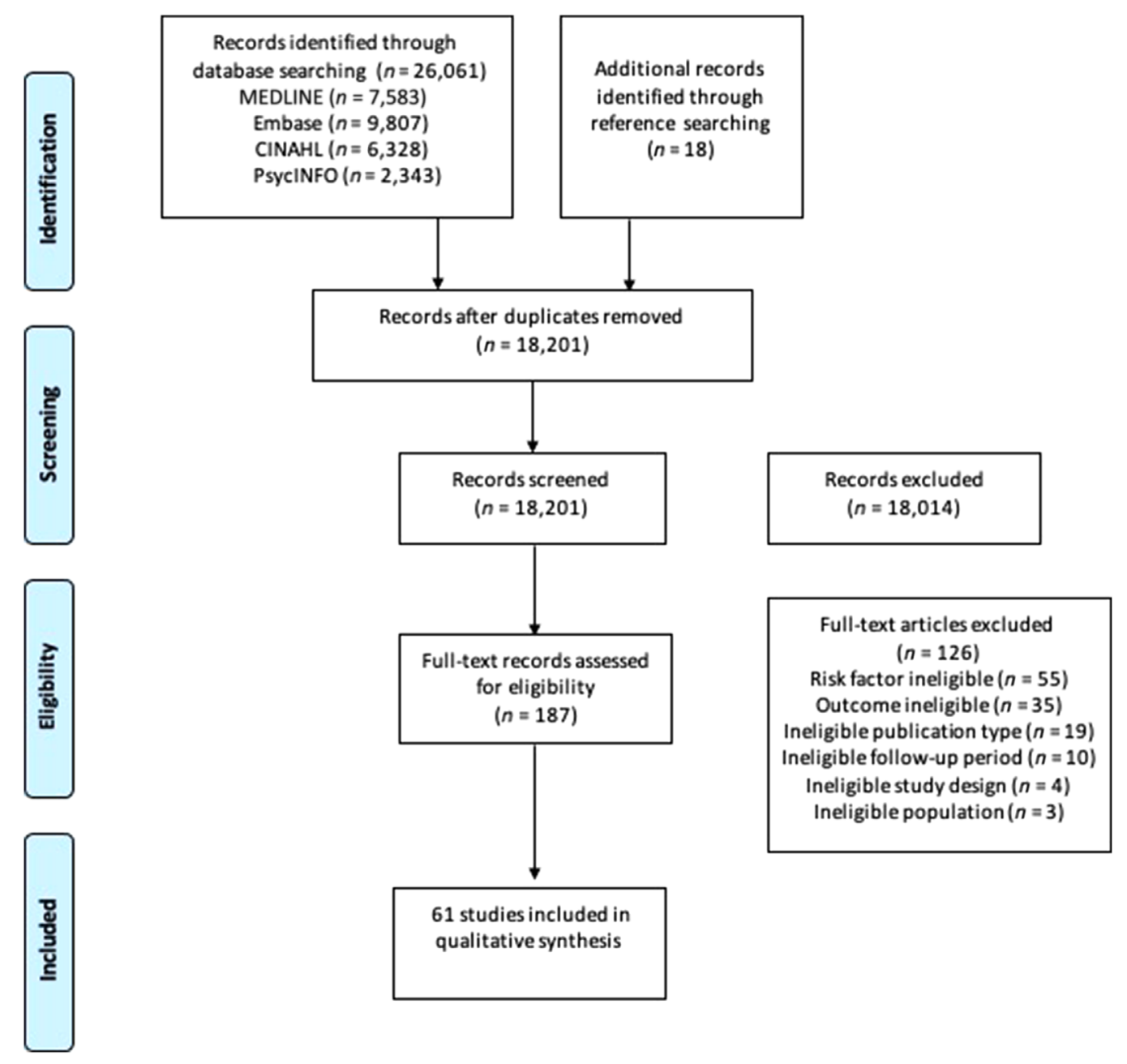

Figure 1 - Preferred Reporting Items for Systematic Reviews and Meta-Analyses flowchart. 
2017; Nilsson, Avlund, \& Lund, 2010; Nilsson, Lund, \& Avlund, 2008; Schroll, Avlund, \& Davidsen, 1997), the Health, Ageing and Body Composition Study (seven studies; Carbone et al., 2013; Duan-Porter et al., 2019; Koster et al., 2005; Lee et al., 2005; Mehta et al., 2007; Thorpe et al., 2011; Visser et al., 2005), and the first Women's Health and Ageing Study (four studies; Brenes et al., 2005; Leveille, Bean, Ngo, McMullen, \& Guralnik, 2007; Leveille et al., 2001; Onder et al., 2003). The majority of studies were based on populations from the United States (27 studies; 11 cohorts) or Finland (16 studies; five cohorts).

Age and sex characteristics. The analyzed sample size of included studies ranged from 136 to 6,981 participants. The mean of the mean ages of participants at baseline was 74.3 years. Mean ages at baseline ranged from 64 to 80 years. The gender distribution of participants varied: nine studies (Brenes et al., 2005; Chaves, Garrett, \& Fried, 2000; Fried, Bandeen-Roche, Chaves, \& Johnson, 2000; Leveille et al., 2001, 2007; Manty et al., 2009; Onder et al., 2003; Viljanen et al., 2012; Weiss, Wolff, Egleston, Seplaki, \& Fried, 2012) (from four cohorts) reported data for women only, and three studies (Avlund et al., 1995; Liljas et al., 2016; van den Brink et al., 2004) (from two cohorts) reported data for men only. Among studies reporting on mixed-gender populations, the proportion of women varied from $46 \%$ to $75 \%$.

Measures of mobility and mobility decline. Thirty-three different measures of mobility were used. The two most commonly used were the Mobility-Help (Mobility-H) scale (11 studies; Avlund, Damsgaard, \& Osler, 2004; Avlund et al., 1995, 2000, 2002; Avlund, Lund, et al., 2004; Avlund, Pedersen, \& Schroll, 2003; Avlund, Vass, \& Hendriksen, 2003; Jørgensen et al., 2017; Nilsson et al., 2008, 2010; Schroll et al., 1997) and the single-item question "Do you have any difficulty walking $1 / 4$ mile/400 m or climbing 1 flight of stairs/10 steps without assistance?" (10 studies; Auais et al., 2018, 2019; Chen, Covinsky, Stijacic Cenzer, Adler, \& Williams, 2012; Deshpande, Metter, Guralnik, Bandinelli, \& Ferrucci, 2014; Eggermont et al., 2014; Koster et al., 2005; Lee et al., 2005; Mehta et al., 2007; Thakral, Shi, Shmerling, Bean, \& Leveille, 2014; Thorpe et al., 2011). Almost all included studies $(59 / 61 ; 97 \%)$ used mobility outcomes that focused solely on walking and/or climbing stairs. Only two studies (Polku et al., 2015; Tsuji, Rantakokko, Portegijs, Viljanen, \& Rantanen, 2018) used the Life Space Assessment which includes ability to move beyond one's neighborhood and/or town by means, such as public transport or driving. There was also variability in the definition of mobility decline. Among studies using the Mobility-H scale, mobility decline was defined in three different ways, most commonly by progression from " 0 activities requiring help" at baseline to "need help in at least one activity" at follow-up (8/11 studies, $73 \%$ ). Mobility decline was measured at a mean of 37 months from recruitment (range $=12-60$ months).

\section{Risk of Bias in Included Studies}

Of the 61 included studies, the 58 etiological and predictor finding studies were appraised using Quality In Prognosis Studies (Supplementary Table S3 [available online]). Risk of bias varied across the studies: 11 studies (Avlund, Vass, \& Hendriksen, 2003; Brenes et al., 2005; Guralnik et al., 1993; Ho, Woo, Yuen, Sham, \& Chan, 1997; Jørgensen et al., 2017; Keskinen, Rantakokko, Suomi, Rantanen, \& Portegijs, 2020; Mehta et al., 2007; Polku et al., 2015; Thorpe et al., 2011; Tsuji et al., 2018; Visser et al., 2005) (11/58, $19 \%$ ) were rated as low risk of bias for all six domains and 30 studies (Abizanda et al., 2013; Auais et al., 2018, 2019; Avlund, Damsgaard, \& Osler, 2004; Avlund et al., 1995, 2000, 2002; Ayis, Gooberman-Hill, Bowling, \& Ebrahim, 2006; Carbone et al., 2013; Deshpande et al., 2014; Duan-Porter et al., 2019; Eggermont et al., 2014; Eronen, von Bonsdorff, Rantakokko, \& Rantanen, 2013; Fried et al., 2000; Koster et al., 2005; LaCroix, Guralnik, Berkman, Wallace, \& Satterfield, 1993; Lang, Llewellyn, Langa, Wallace, \& Melzer, 2008; Leveille et al., 2001; Liljas et al., 2016; Mänty et al., 2007; Manty et al., 2009; Onder et al., 2003; Rantakokko, Iwarsson, Mänty, Leinonen, \& Rantanen, 2012; Rantakokko, Portegijs, Viljanen, Iwarsson, \& Rantanen, 2016; Schroll et al., 1997; Simonsick, Aronson, et al., 2018; Simonsick et al., 2016; Simonsick, Schrack, et al., 2018; Thakral et al., 2014; Weiss et al., 2012) $(30 / 58,52 \%)$ were rated as low risk of bias for five domains. Sources of bias most commonly related to study attrition, specifically lack of details provided regarding reasons for loss to followup or differences in key characteristics between those included and those lost to follow-up. Nine studies (Avlund et al., 2000; Ayis, Bowling, Gooberman-Hill, \& Ebrahim, 2007; Ayis et al., 2006; Eronen et al., 2013; Manty et al., 2009; Onder et al., 2003; Rantakokko et al., 2012; van den Brink et al., 2004; Weiss et al., 2012) $(9 / 58,16 \%)$ were rated as high risk of bias for the study attrition domain, and 33 studies (Abizanda et al., 2013; An \& Lu, 2016; Auais et al., 2018, 2019; Avlund, Damsgaard, \& Osler, 2004; Avlund et al., 2002; Avlund, Lund, et al., 2004; Avlund, Pedersen, \& Schroll, 2003; Carbone et al., 2013; Chen et al., 2012; Crimmins \& Saito, 1993; Deshpande et al., 2014; Duan-Porter et al., 2019; Eggermont et al., 2014; Fried et al., 2000; Guralnik, Ferrucci, Balfour, Volpato, \& Di Iorio, 2001; Koster et al., 2005; LaCroix et al., 1993; Lang et al., 2008; Lee et al., 2005; Leveille et al., 2001, 2007; Lindberg \& Tilvis, 1998; Mänty et al., 2007; Nilsson et al., 2008, 2010; Pine, Gurland, \& Chren, 2000, 2002; Rantakokko et al., 2016; Sauvaget, Tsuji, Aonuma, \& Hisamichi, 1999; Simonsick, Schrack, et al., 2018; Thakral et al., 2014; Viljanen et al., 2012) (33/58, 57\%) were rated as moderate risk of bias. Other major sources of bias related to statistical analysis and reporting, for which four studies (Crimmins \& Saito, 1993; Pine et al., 2002; Simonsick, Aronson, et al., 2018; van den Brink et al., 2004) $(4 / 58,7 \%)$ were rated as high risk of bias, and 10 studies (Avlund, Damsgaard, \& Osler, 2004; Avlund et al., 1995; Avlund, Lund, et al., 2004; Chen et al., 2012; Liljas et al., 2016; Lindberg \& Tilvis, 1998; Pine et al., 2000; Sauvaget et al., 1999; Schroll et al., 1997; Viljanen et al., 2012) (10/58, 17\%) were rated as moderate risk of bias.

The results of the Prediction model Risk of Bias Assessment tool appraisal for the model development studies are presented in Supplementary Table S4 (available online). All three studies (Chaves et al., 2000; Papachristou et al., 2017; Reynolds \& Silverstein, 2003) were judged as having high risk of bias for the analysis domain. This was due to the absence of model performance measures, selection of predictor variables based on univariable analyses, and lack of reporting results of multivariable analyses. All three studies were judged to have both high risk of bias overall and high concern regarding applicability to the review question.

\section{Risk Factors for Mobility Decline Identified in Etiological and Predictor Finding Studies}

A synthesis of the self-reported risk factors for mobility decline examined in etiological and predictor finding studies is presented in Table 1 and Supplementary Table S5 (available online). Due to 


\begin{tabular}{|c|c|c|c|c|c|}
\hline Risk factors & $\begin{array}{l}\text { Number of } \\
\text { participants }^{\mathrm{a}}\end{array}$ & Number of studies examining risk factor & $\begin{array}{l}\text { Number } \\
\text { of } \\
\text { cohorts }\end{array}$ & $\begin{array}{l}\text { Range of } \\
\text { OR } \\
\text { reported }\end{array}$ & $\begin{array}{l}\text { Quality } \\
\text { of } \\
\text { evidence }\end{array}$ \\
\hline \multicolumn{6}{|c|}{ Consistent evidence of increased risk of mobility decline ${ }^{\mathrm{b}}$} \\
\hline Older age & 16,452 & $\begin{array}{l}9 \text { (Auais et al., 2018, 2019; Carbone et al., 2013; Crimmins \& Saito, } \\
\text { 1993; Deshpande et al., 2014; Guralnik et al., 1993, 2001; Ho et al., } \\
\text { 1997; Sauvaget et al., 1999) }\end{array}$ & 7 & $\begin{array}{l}1.1-5.8 \\
>75: 3.5- \\
16.5\end{array}$ & High \\
\hline $\begin{array}{l}\text { Mobility } \\
\text { modification }^{c}\end{array}$ & 1,858 & $\begin{array}{l}5 \text { (Fried et al., 2000; Manty et al., 2009; Pine et al., 2002; } \\
\text { Rantakokko et al., 2016; Weiss et al., 2012) }\end{array}$ & 4 & $\begin{array}{l}2.5-6.2 \\
\mathrm{RR}=5.7- \\
\quad 8.9\end{array}$ & High \\
\hline Widespread pain & 1,102 & 3 (Eggermont et al., 2014; Leveille et al., 2001, 2007) & 2 & $\begin{array}{l}2.5-2.8 \\
\mathrm{RR}=3.6\end{array}$ & Moderate \\
\hline $\begin{array}{l}\text { Gender/sex } \\
\text { (female/women) }\end{array}$ & 14,528 & $\begin{array}{l}8 \text { (Auais et al., 2019; Ayis et al., 2007; Carbone et al., 2013; } \\
\text { Crimmins \& Saito, 1993; Deshpande et al., 2014; Guralnik et al., } \\
\text { 2001; Sauvaget et al., 1999; Schroll et al., 1997) }\end{array}$ & 8 & $1.2-2.6$ & High \\
\hline $\begin{array}{l}\text { Low annual } \\
\text { income }\end{array}$ & 10,047 & 2 (Guralnik et al., 1993; Koster et al., 2005) & 2 & $1.3-2.6$ & Moderate \\
\hline $\begin{array}{l}\text { Low number of } \\
\text { financial assets }\end{array}$ & 6,533 & $\begin{array}{l}5 \text { (Deshpande et al., 2014; Jørgensen et al., 2017; Koster et al., 2005; } \\
\text { Nilsson et al., 2010; Thorpe et al., 2011) }\end{array}$ & 3 & $1.3-1.5$ & High \\
\hline $\begin{array}{l}\text { Low diversity in } \\
\text { social relations }\end{array}$ & 4,308 & 3 (Avlund, Lund, et al., 2004; Ho et al., 1997; Nilsson et al., 2010) & 2 & $1.6-2.8$ & High \\
\hline $\begin{array}{l}\text { Low social } \\
\text { engagement }\end{array}$ & 3,292 & $\begin{array}{l}4 \text { (Avlund, Lund, et al., 2004; Ayis et al., 2006; Jørgensen et al., } \\
\text { 2017; Nilsson et al., 2010) }\end{array}$ & 2 & $1.5-2.9$ & High \\
\hline $\begin{array}{l}\text { Low physical } \\
\text { activity }\end{array}$ & 11,152 & $\begin{array}{l}6 \text { (Abizanda et al., 2013; Avlund et al., 2000; Carbone et al., 2013; } \\
\text { LaCroix et al., 1993; Schroll et al., 1997; Visser et al., 2005) }\end{array}$ & 5 & $\begin{array}{c}1.5-4.3 \\
\mathrm{RR}=4.3\end{array}$ & High \\
\hline $\begin{array}{l}\text { Not walking each } \\
\text { day }\end{array}$ & 4,460 & 2 (Ho et al., 1997; Visser et al., 2005) & 2 & $1.5-2.4$ & Moderate \\
\hline $\begin{array}{l}\text { Greater fatigue } \\
\text { with exertion }\end{array}$ & 1,514 & $\begin{array}{l}3 \text { (Abizanda et al., 2013; Simonsick et al., 2016; Simonsick, Schrack, } \\
\text { et al., 2018) }\end{array}$ & 2 & $1.1-1.5$ & Moderate \\
\hline $\begin{array}{l}\text { Greater tiredness } \\
\text { in daily activities }\end{array}$ & 1,975 & $\begin{array}{l}5 \text { (Avlund et al., 1995, 2002; Avlund, Pedersen, \& Schroll, 2003; } \\
\text { Simonsick et al., 2016; Simonsick, Schrack, et al., 2018) }\end{array}$ & 2 & $1.5-10.8$ & Moderate \\
\hline Hip pain & 2,712 & 2 (Carbone et al., 2013; Eggermont et al., 2014) & 2 & $\begin{array}{c}1.5 \\
\mathrm{RR}=4.5\end{array}$ & Moderate \\
\hline Knee pain & 2,712 & 2 (Carbone et al., 2013; Eggermont et al., 2014) & 2 & $\begin{array}{c}2.0 \\
\mathrm{RR}=2.7\end{array}$ & Moderate \\
\hline Fall/s in past year & 802 & 2 (Carbone et al., 2013; Manty et al., 2009) & 2 & $1.3-3.2$ & Moderate \\
\hline Fear of falling & 1,207 & 2 (Auais et al., 2018; Viljanen et al., 2012) & 2 & $1.1-2.9$ & Moderate \\
\hline $\begin{array}{l}\text { Increasing no. } \\
\text { health conditions }\end{array}$ & 8,723 & 4 (Guralnik et al., 1993, 2001; Ho et al., 1997; Schroll et al., 1997) & 3 & $\begin{array}{c}1.4-3.4 \\
\mathrm{RR}=1.3- \\
5.3\end{array}$ & High \\
\hline Depression & 4,818 & $\begin{array}{l}4 \text { (Ayis et al., 2006; Carbone et al., 2013; Deshpande et al., 2014; Ho } \\
\text { et al., 1997) }\end{array}$ & 4 & $1.1-6.5$ & High \\
\hline Eye conditions & 4,515 & $\begin{array}{l}4 \text { (Ayis et al., 2007; Carbone et al., 2013; Ho et al., 1997; Viljanen } \\
\text { et al., 2012) }\end{array}$ & 4 & $1.6-1.8$ & High \\
\hline Heart conditions & 3,537 & 2 (Crimmins \& Saito, 1993; Ho et al., 1997) & 2 & $1.4-1.7$ & High \\
\hline History of stroke & 10,518 & $\begin{array}{l}4 \text { (Crimmins \& Saito, 1993; Guralnik et al., 1993, 2001; Ho et al., } \\
\text { 1997) }\end{array}$ & 3 & $1.4-2.7$ & Moderate \\
\hline $\begin{array}{l}\text { Sensory } \\
\text { difficulties }\end{array}$ & 2,344 & 2 (Crimmins \& Saito, 1993; Viljanen et al., 2012) & 2 & $1.3-2.5$ & Low \\
\hline $\begin{array}{l}\text { Poor self-rated } \\
\text { health }\end{array}$ & 7,537 & $\begin{array}{l}4 \text { (Avlund et al., 2000; Ayis et al., 2007; Guralnik et al., 2001; Ho } \\
\text { et al., 1997) }\end{array}$ & 4 & $1.3-3.3$ & High \\
\hline $\begin{array}{l}\text { Hospital stay in } \\
\text { past year }\end{array}$ & 4,963 & 2 (Crimmins \& Saito, 1993; Duan-Porter et al., 2019) & 2 & $1.1-1.3$ & Moderate \\
\hline
\end{tabular}




\begin{tabular}{|c|c|c|c|c|c|}
\hline Risk factors & $\begin{array}{l}\text { Number of } \\
\text { participants }^{\mathrm{a}}\end{array}$ & Number of studies examining risk factor & $\begin{array}{c}\text { Number } \\
\text { of } \\
\text { cohorts }\end{array}$ & $\begin{array}{c}\text { Range of } \\
\text { OR } \\
\text { reported }\end{array}$ & $\begin{array}{c}\text { Quality } \\
\text { of } \\
\text { evidence }\end{array}$ \\
\hline \multicolumn{6}{|c|}{ Consistent evidence of no association with mobility decline ${ }^{b}$} \\
\hline $\begin{array}{l}\text { Weight gain in past } \\
12 \text { months }\end{array}$ & 4,415 & 2 (Ho et al., 1997; Lee et al., 2005) & 2 & $\begin{array}{c}0.9 \\
\mathrm{HR}=0.88\end{array}$ & Moderate \\
\hline $\begin{array}{l}\text { Cancer (previous } \\
\text { or current) }\end{array}$ & 7,213 & 3 (Ayis et al., 2007; Guralnik et al., 2001; Ho et al., 1997) & 3 & $0.5-1.3$ & Moderate \\
\hline $\begin{array}{l}\text { Higher no. physi- } \\
\text { cal environment } \\
\text { barriers }\end{array}$ & 1,180 & $\begin{array}{l}4 \text { (Ayis et al., 2006; Eronen et al., 2013; Rantakokko et al., 2012, } \\
\text { 2016) }\end{array}$ & 3 & $0.9-1.5$ & Moderate \\
\hline \multicolumn{6}{|c|}{ Limited evidence of increased risk of mobility decline ${ }^{\mathrm{d}}$} \\
\hline $\begin{array}{l}\text { Housing tenure } \\
\text { (renting) }\end{array}$ & 386 & 1 (Avlund, Lund, et al., 2004) & 1 & 1.9 & Very low \\
\hline $\begin{array}{l}\text { High neighbor- } \\
\text { hood deprivation }\end{array}$ & 3,525 & 1 (Lang et al., 2008) & 1 & 1.8 & Very low \\
\hline $\begin{array}{l}\text { Not having health } \\
\text { insurance }\end{array}$ & 2,622 & 1 (Carbone et al., 2013) & 1 & 1.3 & Very low \\
\hline Not driving a car & 581 & 1 (Tsuji et al., 2018) & 1 & 1.5 & Very low \\
\hline Living alone & 2,825 & $\begin{array}{l}5 \text { (Avlund, Damsgaard, \& Osler, 2004; Avlund et al., 1995; } \\
\text { Jørgensen et al., 2017; Nilsson et al., 2008, 2010; Tsuji et al., 2018) }\end{array}$ & 1 & $1.1-1.7$ & Very low \\
\hline Widowhood & 829 & 1 (van den Brink et al., 2004) & 1 & $1.7-1.8$ & Very low \\
\hline $\begin{array}{l}\text { Currently not } \\
\text { exercising }\end{array}$ & 1,483 & 1 (Ho et al., 1997) & 1 & 2.1 & Very low \\
\hline $\begin{array}{l}\text { Low amount of } \\
\text { exercise per day }\end{array}$ & 1,483 & 1 (Ho et al., 1997) & 1 & $2.2-3.4$ & Very low \\
\hline $\begin{array}{l}\text { Other functional } \\
\text { difficulties }\end{array}$ & 2,145 & 1 (Crimmins \& Saito, 1993) & 1 & $1.3-1.4$ & Very low \\
\hline Low energy level & 579 & 2 (Simonsick et al., 2016; Simonsick, Schrack, et al., 2018) & 1 & $1.1-1.2$ & Very low \\
\hline $\begin{array}{l}\text { Low functional } \\
\text { reserve capacity }\end{array}$ & 427 & 1 (Ayis et al., 2006) & 1 & 6.8 & Very low \\
\hline $\begin{array}{l}\text { Increasing pain } \\
\text { severity }\end{array}$ & 411 & 1 (Eggermont et al., 2014) & 1 & 2.0 & Very low \\
\hline $\begin{array}{l}\text { Increasing pain } \\
\text { interference }\end{array}$ & 411 & 1 (Eggermont et al., 2014) & 1 & 2.4 & Very low \\
\hline $\begin{array}{l}\text { Back pain }+ \text { other } \\
\text { pain }\end{array}$ & 412 & 1 (Eggermont et al., 2014) & 1 & 3.3 & Very low \\
\hline $\begin{array}{l}\text { Hip pain }+ \text { other } \\
\text { pain }\end{array}$ & 412 & 1 (Eggermont et al., 2014) & 1 & 4.8 & Very low \\
\hline $\begin{array}{l}\text { Knee pain }+ \text { other } \\
\text { pain }\end{array}$ & 413 & 1 (Eggermont et al., 2014) & 1 & 3.3 & Very low \\
\hline $\begin{array}{l}\text { Ankle/foot pain }+ \\
\text { other pain }\end{array}$ & 412 & 1 (Eggermont et al., 2014) & 1 & 3.7 & Very low \\
\hline Joint stiffness & 524 & 1 (Thakral et al., 2014) & 1 & $1.3-1.6$ & Very low \\
\hline History of fracture & 2,286 & 2 (Carbone et al., 2013; Ho et al., 1997) & 1 & $1.1-1.6$ & Very low \\
\hline Incontinence & 5,263 & 1 (Guralnik et al., 2001) & 1 & $1.1-1.2$ & Very low \\
\hline Memory loss & 1,483 & 1 (Ho et al., 1997) & 1 & 1.4 & Very low \\
\hline $\begin{array}{l}\text { Respiratory } \\
\text { conditions }\end{array}$ & 491 & 1 (Ayis et al., 2007) & 1 & $1.3-3.5$ & Very low \\
\hline $\begin{array}{l}\text { Health deteriora- } \\
\text { tion in past year }\end{array}$ & 427 & 1 (Ayis et al., 2006) & 1 & 4.3 & Very low \\
\hline $\begin{array}{l}\text { Major life changes } \\
\text { in past } 6 \text { months }\end{array}$ & 491 & 1 (Ayis et al., 2007) & 1 & $1.5-2.3$ & Very low \\
\hline $\begin{array}{l}\text { Low sexual } \\
\text { satisfaction }\end{array}$ & 178 & 1 (Onder et al., 2003) & 1 & 2.6 & Very low \\
\hline
\end{tabular}




\begin{tabular}{|c|c|c|c|c|c|}
\hline Risk factors & $\begin{array}{c}\text { Number of } \\
\text { participants }^{\mathrm{a}}\end{array}$ & Number of studies examining risk factor & $\begin{array}{c}\text { Number } \\
\text { of } \\
\text { cohorts }\end{array}$ & $\begin{array}{c}\text { Range of } \\
\text { OR } \\
\text { reported }\end{array}$ & $\begin{array}{c}\text { Quality } \\
\text { of } \\
\text { evidence }\end{array}$ \\
\hline $\begin{array}{l}\text { Weight change } \\
\text { intention }\end{array}$ & 2,907 & 1 (Lee et al., 2005) & 1 & HR: 1.6 & Very low \\
\hline $\begin{array}{l}\text { Acetaminophen } \\
\text { use }\end{array}$ & 2,486 & 1 (Carbone et al., 2013) & 1 & 1.6 & Very low \\
\hline Antidepressant use & 4,242 & 1 (Carbone et al., 2013) & 1 & 1.1 & Very low \\
\hline NSAID use & 2,486 & 1 (Carbone et al., 2013) & 1 & 1.4 & Very low \\
\hline $\begin{array}{l}\text { Lack of resting } \\
\text { places and long } \\
\text { distances }\end{array}$ & 266 & 1 (Rantakokko et al., 2012) & 1 & $1.9-2.2$ & Very low \\
\hline \multicolumn{6}{|c|}{ Limited evidence of no association with mobility decline ${ }^{\mathrm{d}}$} \\
\hline $\begin{array}{l}\text { Weight change in } \\
\text { past } 12 \text { months }\end{array}$ & 2,932 & 1 (Lee et al., 2005) & 1 & $\begin{array}{c}\mathrm{HR}=0.8- \\
1.49\end{array}$ & Very low \\
\hline $\begin{array}{l}\text { Manual } \\
\text { occupation }\end{array}$ & 243 & 1 (Avlund et al., 2000) & 1 & 1.1 & Very low \\
\hline $\begin{array}{l}\text { Low satisfaction } \\
\text { with living } \\
\text { arrangement }\end{array}$ & 1,483 & 1 (Ho et al., 1997) & 1 & 1.4 & Very low \\
\hline $\begin{array}{l}\text { Low instrumental } \\
\text { support }\end{array}$ & 156 & 1 (Avlund, Lund, et al., 2004) & 1 & 2.8 & Very low \\
\hline $\begin{array}{l}\text { Self-reported } \\
\text { slowing }\end{array}$ & 146 & 1 (Pine et al., 2000) & 1 & 1.0 & Very low \\
\hline $\begin{array}{l}\text { Changes in } \\
\text { tiredness }\end{array}$ & 136 & 1 (Avlund, Vass, \& Hendriksen, 2003) & 1 & 1.6 & Very low \\
\hline Hand/wrist pain & 412 & 1 (Eggermont et al., 2014) & 1 & 1.6 & Very low \\
\hline $\begin{array}{l}\text { Hand/wrist pain }+ \\
\text { other pain }\end{array}$ & 412 & 1 (Eggermont et al., 2014) & 1 & 1.6 & Very low \\
\hline $\begin{array}{l}\text { Lower limb joint } \\
\text { pain }\end{array}$ & 1,483 & 1 (Ho et al., 1997) & 1 & 1.1 & Very low \\
\hline Ankle/foot pain & 413 & 1 (Eggermont et al., 2014) & 1 & 1.7 & Very low \\
\hline $\begin{array}{l}\text { Injury caused by } \\
\text { fall }\end{array}$ & 1,483 & 1 (Ho et al., 1997) & 1 & 1.3 & Very low \\
\hline Angina & 6,981 & 2 (Guralnik et al., 1993, 2001) & 1 & $1.0-1.6$ & Very low \\
\hline Asthma & 1,483 & 1 (Ho et al., 1997) & 1 & 1.1 & Very low \\
\hline Bronchitis & 1,483 & 1 (Ho et al., 1997) & 1 & 1.4 & Very low \\
\hline Claudication & 6,981 & 2 (Guralnik et al., 1993, 2001) & 1 & $1.3-1.4$ & Very low \\
\hline COPD & 5,263 & 1 (Guralnik et al., 2001) & 1 & $1.4-1.8$ & Very low \\
\hline Dyspnea & 6,981 & 2 (Guralnik et al., 1993, 2001) & 1 & $1.2-1.5$ & Very low \\
\hline Headache & 1,483 & 1 (Ho et al., 1997) & 1 & 1.3 & Very low \\
\hline $\begin{array}{l}\text { History of heart } \\
\text { attack }\end{array}$ & 6,981 & 2 (Guralnik et al., 1993, 2001) & 1 & $1.0-1.3$ & Very low \\
\hline $\begin{array}{l}\text { Lack of control } \\
\text { over life }\end{array}$ & 456 & 1 (Ayis et al., 2007) & 1 & $1.0-1.9$ & Very low \\
\hline $\begin{array}{l}\text { Low satisfaction } \\
\text { with life }\end{array}$ & 1,483 & 1 (Ho et al., 1997) & 1 & 1.2 & Very low \\
\hline $\begin{array}{l}\text { Increased vulnera- } \\
\text { bility to accidents }\end{array}$ & 491 & 1 (Ayis et al., 2007) & 1 & $1.3-2.0$ & Very low \\
\hline $\begin{array}{l}\text { Increased vulnera- } \\
\text { bility to mugging, } \\
\text { burglary }\end{array}$ & 491 & 1 (Ayis et al., 2007) & 1 & $0.8-1.9$ & Very low \\
\hline Estrogen use & 2,486 & 1 (Carbone et al., 2013) & 1 & 0.8 & Very low \\
\hline Vitamin D use & 2,486 & 1 (Carbone et al., 2013) & 1 & 0.8 & Very low \\
\hline $\begin{array}{l}\text { Distance to park or } \\
\text { other green area }\end{array}$ & 261 & 1 (Eronen et al., 2013) & 1 & 2.3 & Very low \\
\hline
\end{tabular}




\begin{tabular}{|c|c|c|c|c|c|}
\hline Risk factors & $\begin{array}{l}\text { Number of } \\
\text { participants }^{\mathrm{a}}\end{array}$ & Number of studies examining risk factor & $\begin{array}{l}\text { Number } \\
\text { of } \\
\text { cohorts }\end{array}$ & $\begin{array}{l}\text { Range of } \\
\text { OR } \\
\text { reported }\end{array}$ & $\begin{array}{l}\text { Quality } \\
\text { of } \\
\text { evidence }\end{array}$ \\
\hline $\begin{array}{l}\text { Distance to out- } \\
\text { door recreational } \\
\text { facilities }\end{array}$ & 261 & 1 (Eronen et al., 2013) & 1 & 1.7 & Very low \\
\hline $\begin{array}{l}\text { Perceived hilliness } \\
\text { in neighborhood }\end{array}$ & 551 & 1 (Keskinen et al., 2020) & 1 & 1.7 & Very low \\
\hline $\begin{array}{l}\text { Hilly terrain and } \\
\text { streets in poor } \\
\text { condition }\end{array}$ & 266 & 1 (Rantakokko et al., 2012) & 1 & $1.1-1.4$ & Very low \\
\hline $\begin{array}{l}\text { Noisy traffic and } \\
\text { dangerous } \\
\text { crossroads }\end{array}$ & 266 & 1 (Rantakokko et al., 2012) & 1 & $1.3-1.5$ & Very low \\
\hline \multicolumn{6}{|c|}{ Inconsistent/unclear association with mobility decline $\mathrm{e}^{\mathrm{e}}$} \\
\hline Race (non-White) & 4,784 & $\begin{array}{l}3 \text { (Carbone et al., 2013; Crimmins \& Saito, 1993; Thorpe et al., } \\
\text { 2011) }\end{array}$ & 2 & $0.9-3.0$ & Very low \\
\hline $\begin{array}{l}\text { High alcohol } \\
\text { consumption }\end{array}$ & 8,464 & 2 (Ho et al., 1997; LaCroix et al., 1993) & 2 & $1.0-1.2$ & Very low \\
\hline Smoker/ex-smoker & 8,707 & $\begin{array}{l}4 \text { (Avlund et al., 2000; Guralnik et al., 2001; Ho et al., 1997; LaCroix } \\
\text { et al., 1993) }\end{array}$ & 3 & $0.8-3.6$ & Very low \\
\hline $\begin{array}{l}\text { BMI outside of } \\
\text { normal range }\end{array}$ & 14,924 & $\begin{array}{l}8 \text { (Carbone et al., 2013; Crimmins \& Saito, 1993; Deshpande et al., } \\
\text { 2014; Guralnik et al., 2001; Ho et al., 1997; LaCroix et al., 1993; } \\
\text { Lindberg \& Tilvis, 1998; Tsuji et al., 2018) }\end{array}$ & 7 & $0.8-2.1$ & Very low \\
\hline $\begin{array}{l}\text { Weight loss in past } \\
12 \text { months }\end{array}$ & 5,350 & 3 (Abizanda et al., 2013; Ho et al., 1997; Lee et al., 2005) & 3 & $\begin{array}{l}1.0-1.6 \\
\mathrm{HR}=0.8 \\
\quad 2.0\end{array}$ & Very low \\
\hline $\begin{array}{l}\text { Low level of } \\
\text { education }\end{array}$ & 12,996 & $\begin{array}{l}11 \text { (Avlund, Damsgaard, \& Osler, 2004; Avlund et al., 1995, 2000; } \\
\text { Ayis et al., 2007; Carbone et al., 2013; Deshpande et al., 2014; } \\
\text { Guralnik et al., 1993, 2001; Ho et al., 1997; Koster et al., 2005; } \\
\text { Thorpe et al., 2011) }\end{array}$ & 6 & $0.9-2.3$ & Very low \\
\hline $\begin{array}{l}\text { Income from pen- } \\
\text { sion only }\end{array}$ & 1,869 & $\begin{array}{l}3 \text { (Avlund, Damsgaard, \& Osler, 2004; Avlund et al., 1995; Ho et al., } \\
\text { 1997) }\end{array}$ & 2 & $1.1-3.1$ & Very low \\
\hline Low social status & 2,789 & 2 (Avlund et al., 1995; Chen et al., 2012) & 2 & $1.0-1.5$ & Very low \\
\hline Low social support & 6,082 & $\begin{array}{l}5 \text { (Avlund, Lund, et al., 2004; Ayis et al., 2006; Carbone et al., 2013; } \\
\text { Deshpande et al., 2014; Jørgensen et al., 2017) }\end{array}$ & 4 & $0.9-2.3$ & Very low \\
\hline $\begin{array}{l}\text { Higher no. mus- } \\
\text { culoskeletal } \\
\text { conditions }\end{array}$ & 734 & 2 (Avlund et al., 2000; Ayis et al., 2007) & 2 & $1.8-2.8$ & Very low \\
\hline Back pain & 1,077 & 2 (Eggermont et al., 2014; Simonsick, Aronson, et al., 2018) & 2 & $1.0-1.7$ & Very low \\
\hline Hip fracture & 7,408 & 2 (Crimmins \& Saito, 1993; Guralnik et al., 2001) & 2 & $1.0-3.2$ & Very low \\
\hline Anxiety & 4,209 & 3 (Ayis et al., 2006; Brenes et al., 2005; Mehta et al., 2007) & 3 & $\begin{array}{c}1.0-1.2 \\
\mathrm{HR}=1.3\end{array}$ & Very low \\
\hline Arthritis & 10,609 & $\begin{array}{l}4 \text { (Crimmins \& Saito, 1993; Guralnik et al., 1993, 2001; Ho et al., } \\
\text { 1997) }\end{array}$ & 3 & $1.1-1.4$ & Very low \\
\hline $\begin{array}{l}\text { Circulatory } \\
\text { conditions }\end{array}$ & 491 & 1 (Ayis et al., 2007) & 1 & 1.7 & Very low \\
\hline Cognitive decline & 6,376 & 3 (Ayis et al., 2007; Deshpande et al., 2014; Guralnik et al., 2001) & 3 & $1.0-2.5$ & Very low \\
\hline Diabetes & 8,464 & 3 (Guralnik et al., 1993, 2001; Ho et al., 1997) & 2 & $1.3-2.3$ & Very low \\
\hline $\begin{array}{l}\text { Dizziness/balance } \\
\text { difficulties }\end{array}$ & 1,773 & 2 (Ho et al., 1997; Viljanen et al., 2012) & 2 & $1.0-1.4$ & Very low \\
\hline Hearing problems & 5,837 & $\begin{array}{l}6 \text { (Ayis et al., 2006, 2007; Ho et al., 1997; Liljas et al., 2016; Polku } \\
\text { et al., 2015; Viljanen et al., 2012) }\end{array}$ & 5 & $1.1-2.8$ & Very low \\
\hline $\begin{array}{l}\text { High blood } \\
\text { pressure }\end{array}$ & 10,609 & 3 (Crimmins \& Saito, 1993; Guralnik et al., 1993; Ho et al., 1997) & 3 & $1.1-1.2$ & Very low \\
\hline
\end{tabular}

Note. OR of $1.4-2.5=$ small effect; $2.5-4.25=$ moderate effect; $\geq 4.25=$ large effect. The range shown is the lowest and highest OR reported by included studies. $\mathrm{RR}=$ risk ratio; $\mathrm{HR}=$ hazard ratio; GRADE $=$ Grading of Recommendations Assessment, Development and Evaluation; $\mathrm{OR}=$ odds ratio; $\mathrm{BMI}=$ body mass index; NSAID = nonsteroidal anti-inflammatory drug; $\mathrm{COPD}=$ chronic obstructive pulmonary disease.

${ }^{\mathrm{a}}$ When more than one study examining the same factor was derived from the same cohort, the highest number of participants was added to the participant total. ${ }^{\mathrm{b}}$ Two or more studies from two or more cohorts; $>75 \%$ of studies showing the same direction of effect. ${ }^{\circ}$ Mobility modifications defined as any of resting during the task, using an aid, using handrails, reduced frequency, or reduced speed of performing the task. ${ }^{\mathrm{d}}$ Single study or multiple studies from single cohort. ${ }^{\mathrm{e}}$ Two or more studies from two or more cohorts; $<75 \%$ of studies showing the same direction of effect, or single study with insufficient data to assess association. 
variation in methods of measurement for risk factors and/or mobility outcomes, we were unable to formally pool the results using meta-analysis (Riley et al., 2019).

Of the 107 risk factors examined in these studies, there was consistent evidence (less than or equal to two studies from less than or equal to two cohorts) that 24 factors were associated with mobility decline. Of these 24 , three were associated with a moderate/large effect and 21 associated with a small effect. There was also consistent evidence of no association with mobility decline for three factors. The results and discussion will focus on these 27 factors.

Among the remaining 80 risk factors, there was limited evidence (single study or multiple studies from a single cohort) of association with mobility decline for 30 , limited evidence of no effect on mobility decline for 30, and inconsistent or unclear effects on mobility decline for 20 factors (Table 1 and Supplementary Table S5 [available online]).

Factors with consistent moderate/large effect on mobility decline. Three factors were consistently associated with mobility decline with moderate-large effect sizes. There was high quality evidence for older age, with large effect sizes reported for participants aged 75 years or older compared to those younger than 75 years. There was also high quality evidence and a dose-response effect observed for mobility modifications (resting during the task, using an aid, using handrails, reduced frequency or reduced speed of performing the task). Moderate quality evidence was found for the presence of widespread pain as a risk factor for mobility decline.

Factors with consistent small effect on mobility decline. For the remaining 21 risk factors consistently associated with mobility decline, the strength of the association represented by ORs was considered small (Table 1). There was high-quality evidence for five demographic and socioeconomic risk factors. Female gender was associated with increased risk of mobility decline, as was low annual income; low number of financial assets; low diversity in social relations; and low social engagement.

Five risk factors were associated with physical- and fatiguerelated issues. Low physical activity was associated with mobility decline with high quality evidence. There was moderate quality evidence for not walking every day, having at least one fall in the past year, higher fatigue after exertion, and greater tiredness in daily activities as risk factors for mobility decline.

Evidence for the consistent small effect of 10 health factors on mobility decline ranged from high to low quality. High quality evidence was found for increasing number of health conditions. Specific health conditions presenting as risk factors for mobility decline included depression (high quality evidence), eye conditions (high quality evidence), heart conditions (high quality evidence), history of stroke (moderate quality evidence), and sensory difficulties (low quality evidence). Other factors that showed a consistent small association with increased risk of mobility decline were hip pain (moderate quality evidence), knee pain (moderate quality evidence), poor self-rated health (high quality evidence), and reporting a hospital stay in the past year (Table 1 and Supplementary Table S5 [available online]).

\section{Factors with consistent lack of effect on mobility decline.}

Moderate quality evidence was found for three factors which consistently demonstrated no association with increased risk of mobility decline: weight gain in the past 12 months, history of or current cancer diagnosis, and reporting a higher number of physical environment barriers (e.g., long distances, busy traffic, rough or hilly terrain, high crime area; Table 1 and Supplementary Table S5 [available online]).

\section{Model Development Studies}

The three model development studies are summarized in Table 2 and Supplementary Table S2 (available online). Follow-up periods

\section{Table 2 Synthesis of Risk Factors for Mobility Decline Examined in Included Model Development Studies}

\begin{tabular}{|c|c|c|c|c|c|}
\hline Study & Outcome & $\begin{array}{l}\text { Risk factor/s } \\
\text { included in final } \\
\text { model }\end{array}$ & $\begin{array}{l}\text { Study } \\
\text { sample size } \\
n \text { (\% of } \\
\text { participants with } \\
\text { the outcome) }\end{array}$ & $\begin{array}{l}\text { Type of } \\
\text { validation }\end{array}$ & Performance \\
\hline $\begin{array}{l}\text { Chaves et al. } \\
(2000)\end{array}$ & $\begin{array}{l}\text { Onset of mobility difficulty. Diffi- } \\
\text { culty in at least one of the tasks: } \\
\text { walking } 0.8 \mathrm{~km} \text {; climbing } 10 \mathrm{steps} \\
\text { transferring from/into a car or bus }\end{array}$ & $\begin{array}{l}\text { Task modification }+ \\
\text { walking time for } 1 \mathrm{~m} \\
\text { at usual pace }+ \text { one- } \\
\text { leg stance balance }\end{array}$ & $266(23.9)$ & $\begin{array}{l}\text { Bootstrap } \\
\text { validation }\end{array}$ & $\begin{array}{l}\text { Apparent performance ONLY } \\
\text { - AUC }=0.73 \\
\text { - Classification and discrimination } \\
\text { measures (correct classification }{ }^{\text {a) }} \text { ) } \\
\text { were presented using multiple } \\
\text { predicted probability thresholds }\end{array}$ \\
\hline $\begin{array}{l}\text { Papachristou } \\
\text { et al. (2017) }\end{array}$ & $\begin{array}{l}\text { Incident disability. Difficulty in at } \\
\text { least one of the tasks: walking } \\
400 \text { yards; going up or down stairs }\end{array}$ & $\begin{array}{l}\text { Age }+ \text { slow walking } \\
\text { speed }+ \text { physical } \\
\text { inactive }+ \text { exhaustion }\end{array}$ & NR (15.0) & $\begin{array}{l}\text { Not } \\
\text { reported }\end{array}$ & - $\mathrm{AUC}=0.68(0.63-0.72)$ \\
\hline $\begin{array}{l}\text { Reynolds and } \\
\text { Silverstein } \\
(2003)\end{array}$ & Onset of walking disability & $\begin{array}{l}41 \text { variables includ- } \\
\text { ing demographic, } \\
\text { medical, and behav- } \\
\text { ioral characteristics }\end{array}$ & $3,964(10.7)$ & $\begin{array}{l}\text { Not } \\
\text { reported }\end{array}$ & $\begin{array}{l}\text { Apparent performance ONLY: } \\
\text { - AUC }=0.819 \\
\text { - Hosmer-Lemeshow test }=14.47 \text {, } \\
\quad p=.07 \\
\text { - Adjusted } R^{2}=.1817\end{array}$ \\
\hline
\end{tabular}

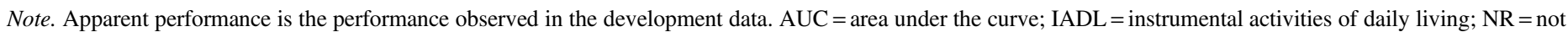
reported.

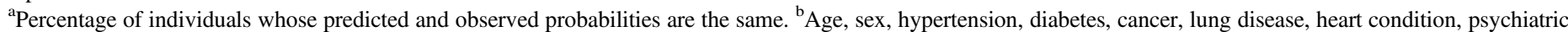

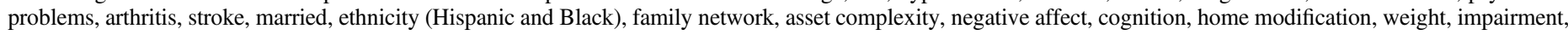

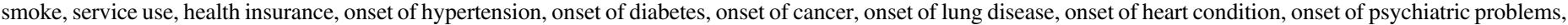

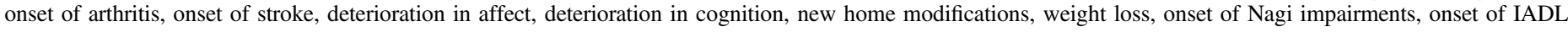
impairments, smoking less, new service use, vigorous exercise, and preventive test. 
varied from recruitment: 18 months (Chaves et al., 2000); 36 months (Papachristou et al., 2017); and 60 months (Reynolds \& Silverstein, 2003). Overall, the three models showed a moderate discriminative ability (area under curve $[\mathrm{AUC}]=0.68-0.81$ ), but calibration measures were poorly reported or missing. Only one model was internally validated (Chaves et al., 2000) and none undertook external model validation.

\section{Discussion}

This systematic review provides evidence on self-reported factors which predict decline in self-reported mobility between 12 months and 5 years in older adults. Among the 107 risk factors examined in the included studies, we found that 24 had consistent moderate or high quality evidence indicating their association with mobility decline. Effect sizes were generally small, with moderate/large effect sizes observed for three factors: older age ( $\geq 75$ years), widespread pain, and mobility modifications. Three existing model development studies were identified, all demonstrating moderate model discriminatory performance, and limited by high risk of bias because of analytical shortcomings.

To our knowledge, this is the first systematic review to comprehensively summarize available evidence about self-reported risk factors for self-reported mobility decline among older adults. Among the 107 risk factors we reviewed, only one quarter had sufficient evidence from which to draw firm conclusions regarding association with mobility decline. This does not mean that the remaining factors do not play a role in mobility decline in later life, rather that evidence was insufficient to draw such a conclusion. Significant heterogeneity across studies in definitions and assessments of risk factors and mobility decline precluded quantitative synthesis of the data, and consequently our findings must be interpreted with caution.

A 2008 narrative review concluded that the association between older age and increased mobility limitations was statistically significant over 70 years of age (Yeom et al., 2008). Agerelated changes in mobility may result from physiological changes, such as decreased muscle strength and power, reduced bone mass, and decreased response to balance perturbations (Byrne, Faure, Keene, \& Lamb, 2016). However, age-related changes in mobility may also be associated with, or accelerated by, lack of physical activity, leading to a negative cycle of decline (Brach et al., 2003).

The negative effects of widespread pain on physical functions, including mobility, are well established (Butera, Roff, Buford, \& CruzAlmeida, 2019). However, the pathway from widespread pain to mobility limitation is not clear. Nagi's disablement model suggests that widespread pain may lead to decreased physical activity, and in turn loss of muscle strength and the development of mobility limitations (Verbrugge \& Jette, 1994). However, Leveille et al. concluded that pain is a unique domain as a cause of mobility difficulty, independent of the usual pathway via physical impairments (Leveille et al., 2007).

Mobility modifications, such as resting, using an aid, using handrails, reduced frequency, or speed of performing a task are important risk factors among older adults as they have been identified as "preclinical mobility decline" (Fried et al., 2000; Rantakokko et al., 2013). Making such modifications may compensate for the impact of underlying health changes, such as pain, reduced muscle strength, or fatigue on mobility (Fried et al., 2000). An individual's transition to mobility difficulty easily occurs when any internal or external changes overwhelm the compensations being employed. Our findings reiterate the importance of assessing the use of mobility modifications to identify people at high risk of mobility decline so the underlying causes can be addressed.
This review identifies several opportunities for targeting modifiable factors associated with mobility decline, which should be considered in management of older adults at risk of mobility decline, and intervention development. We found high quality evidence of the association between two social factors (low diversity in social relations and low social engagement) and increased risk of mobility decline. This highlights the importance of assessing social outcomes, which are often neglected, and the potential of social prescribing interventions. We also found high quality evidence for low physical activity as a risk factor for mobility decline. Many physical activity programs have been used to improve mobility in older adults, with positive effects (Rantakokko et al., 2013). Assessing and addressing fears of falling in older adults is also important, as indicated by our finding of moderate quality evidence for fear of falling as a risk factor for mobility decline.

Our findings highlight the complexity of managing older adults at risk of mobility decline. Mobility decline in older age is multifactorial, and many older adults may report multiple risk factors concurrently. Combinations of multiple risk factors may have greater impact than the sum of their individual effects, and future research should explore this. Future interventions targeting maintenance of mobility should be multidimensional, combine interventions for multiple risk factors, and control for confounding factors. Our findings also highlight the limited evidence for existing prognostic models for mobility decline. These results may guide future longitudinal studies to develop a prediction instrument for practical application.

\section{Limitations of the Review}

Despite the authors' effort to construct a sensitive search strategy, there is a chance that relevant studies were overlooked. Of the included studies, 70\% (43/61) came from two countries (United States and Finland), which may impact the generalizability of findings to other populations. Only 3\% of studies (2/61) included participants from low-income countries (World Bank, 2020). None of the included studies were conducted in Africa, and only two were conducted in Asia (Hong Kong and Japan). Differing lifestyles and cultures may mean that risk factors are not the same in these settings, and findings should not be generalized. Studies in more diverse international settings are warranted. Grouping together everyone with mobility decline may dilute associations linked with specific subtypes of mobility decline, such as gradual versus rapid decline. Analysis of risk factors according to definitions of mobility decline may give more insight into causal pathways. Almost all included studies $(59 / 61,97 \%)$ used narrow measures of mobility, focusing on ability to walk and/or climb stairs. Use of broader measures of mobility, such as life-space assessment may enable identification of additional risk factors, and/or which risk factors are associated with different aspects of mobility. Finally, by excluding studies with follow-up periods of $<12$ months or $>5$ years, there is a risk that studies showing short- or long-term results were missed.

\section{Conclusion}

In conclusion, our systematic review identified 24 wide-ranging self-reported risk factors consistently associated with self-reported mobility decline in older people. Older age beyond 75 years, the presence of widespread pain, and mobility modifications were the risk factors with the highest effect.

\section{Acknowledgments}

The authors would like to express their gratitude to those study authors who responded to study queries and data requests. 
Nicolson and Sanchez-Santos are joint first authors. PN, MS, JB, SK, LW, EW, and SL made substantial contributions to the conception and design of this review; PN, MS, and SK acquired the data; PN, MS, JB, EW, and SL completed analysis and interpretation of the data. PN and MS wrote the first draft of this article, and all authors contributed to revising it critically for important intellectual content. All authors gave their final approval of the version to be published.

The authors do not have any conflicts of interest to declare. SL and EW receive funding from the Collaboration for Leadership in Applied Health Research and Care Oxford at Oxford Health NHS Foundation Trust, and SL, MS, and EW are supported by NIHR Musculoskeletal Biomedical Research Centre, Oxford. PN is funded by a Versus Arthritis Foundation Fellowship (Grant Reference Number 22428). JB is supported by NIHR Research Capability Funding via University Hospitals Coventry and Warwickshire. SK is funded by Cancer Research UK (Grant C49297/A27294). The funders had no role in the design, collection, analysis, or reporting of this review. The views expressed are those of the authors and not necessarily those of the NHS, the NIHR or the Department of Health and Social Care.

\section{References}

Abizanda, P., Romero, L., Sánchez-Jurado, P.M., Martinez-Reig, M., Gomez-Arnedo, L., \& Alfonso, S.A. (2013). Frailty and mortality, disability and mobility loss in a Spanish cohort of older adults: The FRADEA study. Maturitas, 74(1), 54-60. PubMed ID: 23107816 doi:10.1016/j.maturitas.2012.09.018

Alexander, N.B., Guire, K.E., Thelen, D.G., Ashton-Miller, J.A., Schultz, A.B., Grunawalt, J.C., \& Giordani, B. (2000). Self-reported walking ability predicts functional mobility performance in frail older adults. Journal of the American Geriatrics Society, 48(11), 1408-1413. PubMed ID: 11083316 doi:10.1111/j.1532-5415.2000.tb02630.x

An, R., \& Lu, L. (2016). Antidepressant use and functional limitations in U.S. older adults. Journal of Psychosomatic Research, 80, 31-36. PubMed ID: 26721545 doi:10.1016/j.jpsychores.2015.11.007

Auais, M., Ahmed, T., Alvarado, B., Phillips, S.P., Rosendaal, N., Curcio, C.L., . . Z Zunzunegui, M.V. (2019). Gender differences in four-year incidence of self-reported and performance-based functional disability: The international mobility in aging study. Archives of Gerontology and Geriatrics, 82, 266-272. PubMed ID: 30878824 doi:10. 1016/j.archger.2019.03.002

Auais, M., French, S., Alvarado, B., Pirkle, C., Belanger, E., \& Guralnik, J. (2018). Fear of falling predicts incidence of functional disability 2 years later: A perspective from an international cohort study. The Journals of Gerontology. Series A, Biological Sciences \& Medical Sciences, 73(9), 1212-1215. PubMed ID: 29220420 doi:10.1093/ gerona/glx 237

Avlund, K., Damsgaard, M.T., \& Osler, M. (2004). Social position and functional decline among non-disabled old men and women. European Journal of Public Health, 14(2), 212-216. PubMed ID: 15230514 doi:10.1093/eurpub/14.2.212

Avlund, K., Damsgaard, M.T., Sakari-Rantala, R., Laukkanen, P., \& Schroll, M. (2002). Tiredness in daily activities among nondisabled old people as determinant of onset of disability. Journal of Clinical Epidemiology, 55(10), 965-973. PubMed ID: 12464372 doi:10.1016/ S0895-4356(02)00463-8

Avlund, K., Davidsen, M., \& Schultz-Larsen, K. (1995). Changes in functional ability from ages 70 to 75 . A Danish longitudinal study. Journal of Aging and Health, 7(2), 254-282. PubMed ID: 10165956 doi:10.1177/089826439500700205

Avlund, K., Lund, R., Holstein, B.E., \& Due, P. (2004). Social relations as determinant of onset of disability in aging. Archives of Gerontology and Geriatrics, 38(1), 85-99. PubMed ID: 14599708 doi:10.1016/j. archger.2003.08.003

Avlund, K., Osler, M., Damsgaard, M.T., Christensen, U., \& Schroll, M. (2000). The relations between musculoskeletal diseases and mobility among old people: Are they influenced by socio-economic, psychosocial, and behavioral factors? International Journal of Behavioral Medicine, 7(4), 322-339. doi:10.1207/S15327558IJBM0704_04

Avlund, K., Pedersen, A.N., \& Schroll, M. (2003). Functional decline from age 80 to 85 : Influence of preceding changes in tiredness in daily activities. Psychosomatic Medicine, 65(5), 771-777. PubMed ID: 14508019 doi:10.1097/01.PSY.0000082640.61645.BF

Avlund, K., Vass, M., \& Hendriksen, C. (2003). Onset of mobility disability among community-dwelling old men and women. The role of tiredness in daily activities. Age and Ageing, 32(6), 579-584. PubMed ID: 14599997 doi:10.1093/ageing/afg101

Ayis, S., Gooberman-Hill, R., Bowling, A., \& Ebrahim, S. (2006). Predicting catastrophic decline in mobility among older people. Age and Ageing, 35(4), 382-387. PubMed ID: 16638760 doi:10. 1093/ageing/afl004

Ayis, S.A., Bowling, A., Gooberman-Hill, R., \& Ebrahim, S. (2007). The effect of definitions of activities of daily living on estimates of changing ability among older people. International Journal of Rehabilitation Research, 30(1), 39-46. PubMed ID: 17293719 doi:10. 1097/MRR.0b013e328013dafb

Brach, J.S., FitzGerald, S., Newman, A.B., Kelsey, S., Kuller, L., VanSwearingen, J.M., \& Kriska, A.M. (2003). Physical activity and functional status in community-dwelling older women: A 14-year prospective study. Archives of Internal Medicine, 163(21), 2565-2571. PubMed ID: 14638556 doi:10.1001/archinte.163.21.2565

Brenes, G.A., Guralnik, J.M., Williamson, J.D., Fried, L.P., Simpson, C., Simonsick, E.M., \& Penninx, B.W. (2005). The influence of anxiety on the progression of disability. Journal of the American Geriatrics Society, 53(1), 34-39. PubMed ID: 15667373 doi:10.1111/j.15325415.2005.53007.x

Butera, K.A., Roff, S.R., Buford, T.W., \& Cruz-Almeida, Y. (2019). The impact of multisite pain on functional outcomes in older adults: Biopsychosocial considerations. Journal of Pain Research, 12, 1115-1125. PubMed ID: 30992680 doi:10.2147/JPR.S192755

Byrne, C., Faure, C., Keene, D.J., \& Lamb, S.E. (2016). Ageing, muscle power and physical function: A systematic review and implications for pragmatic training interventions. Sports Medicine, 46(9), 1311-1332. PubMed ID: 26893098 doi:10.1007/s40279-0160489-x

Carbone, L.D., Satterfield, S., Liu, C., Kwoh, K.C., Neogi, T., Tolley, E., \& Nevitt, M. (2013). Assistive walking device use and knee osteoarthritis: Results from the health, aging and body composition study (health ABC study). Archives of Physical Medicine and Rehabilitation, 94(2), 332-339. PubMed ID: 23041146 doi:10.1016/j.apmr. 2012.09.021

Chaves, P.H., Garrett, E.S., \& Fried, L.P. (2000). Predicting the risk of mobility difficulty in older women with screening nomograms: The women's health and aging study II. Archives of Internal Medicine, 160(16), 2525-2533. PubMed ID: 10979066 doi:10.1001/archinte. 160.16 .2525

Chen, B., Covinsky, K.E., Stijacic Cenzer, I., Adler, N., \& Williams, B.A. (2012). Subjective social status and functional decline in older adults. Journal of General Internal Medicine, 27(6), 693-699. PubMed ID: 22215272 doi:10.1007/s11606-011-1963-7

Chinn, S. (2000). A simple method for converting an odds ratio to effect size for use in meta-analysis. Statistics in Medicine, 19(22), 31273131. PubMed ID: 11113947 doi:10.1002/1097-0258(20001130)19: 22<3127::AID-SIM784>3.0.CO;2-M 
Chung, J., Demiris, G., \& Thompson, H.J. (2015). Instruments to assess mobility limitation in community-dwelling older adults: A systematic review. Journal of Aging and Physical Activity, 23(2), 298-313. PubMed ID: 24589525 doi:10.1123/japa.2013-0181

Crimmins, E.M., \& Saito, Y. (1993). Getting better and getting worse: Transitions in functional status among older Americans. Journal of Aging and Health, 5(1), 3-36. doi:10.1177/089826439300500101

Deshpande, N., Metter, J.E., Guralnik, J., Bandinelli, S., \& Ferrucci, L. (2014). Sensorimotor and psychosocial determinants of 3-year incident mobility disability in middle-aged and older adults. Age and Ageing, 43(1), 64-69. PubMed ID: 24042004 doi:10.1093/ageing/aft135

Duan-Porter, W., Vo, T.N., Ullman, K., Langsetmo, L., Strotmeyer, E.S., Taylor, B.C., . . . Ensrud, K.E. (2019). Hospitalization-associated change in gait speed and risk of functional limitations for older adults. The Journals of Gerontology. Series A, Biological Sciences \& Medical Sciences, 74(10), 1657-1663. PubMed ID: 30715162 doi:10.1093/gerona/glz027

Eggermont, L.H., Leveille, S.G., Shi, L., Kiely, D.K., Shmerling, R.H., Jones, R.N., . . Bean, J.F. (2014). Pain characteristics associated with the onset of disability in older adults: The maintenance of balance, independent living, intellect, and zest in the Elderly Boston Study. Journal of the American Geriatric Society, 62(6), 1007-1016. PubMed ID: 24823985 doi:10.1111/jgs. 12848

Eronen, J., von Bonsdorff, M., Rantakokko, M., \& Rantanen, T. (2013). Environmental facilitators for outdoor walking and development of walking difficulty in community-dwelling older adults. European Journal of Ageing, 11(1), 67-75. PubMed ID: 28804315 doi:10. 1007/s10433-013-0283-7

Fried, L.P., Bandeen-Roche, K., Chaves, P.H., \& Johnson, B.A. (2000). Preclinical mobility disability predicts incident mobility disability in older women. The Journals of Gerontology. Series A, Biological Sciences \& Medical Sciences, 55(1), M43-M52. PubMed ID: 10719772 doi:10.1093/gerona/55.1.M43

Guralnik, J.M., Ferrucci, L., Balfour, J.L., Volpato, S., \& Di Iorio, A. (2001). Progressive versus catastrophic loss of the ability to walk: Implications for the prevention of mobility loss. Journal of the American Geriatric Society, 49(11), 1463-1470. PubMed ID: 11890584 doi:10.1046/j.1532-5415.2001.4911238.x

Guralnik, J.M., LaCroix, A.Z., Abbott, R.D., Berkman, L.F., Satterfield, S., Evans, D.A., \& Wallace, R.B. (1993). Maintaining mobility in late life. I. Demographic characteristics and chronic conditions. American Journal of Epidemiology, 137(8), 845-857. PubMed ID: 8484376 doi:10.1093/oxfordjournals.aje.a116746

Hayden, J.A., Tougas, M.E., Riley, R., Iles, R., \& Pincus, T. (2014). Individual recovery expectations and prognosis of outcomes in nonspecific low back pain: Prognostic factor exemplar review. Cochrane Database of Systematic Reviews, 2014(9), CD011284. doi:10.1002/ 14651858.cd011284

Hayden, J.A., van der Windt, D.A., Cartwright, J.L., Cote, P., \& Bombardier, C. (2013). Assessing bias in studies of prognostic factors. Annals of Internal Medicine, 158(4), 280-286. PubMed ID: 23420236 doi:10. 7326/0003-4819-158-4-201302190-00009

Ho, S.C., Woo, J., Yuen, Y.K., Sham, A., \& Chan, S.G. (1997). Predictors of mobility decline: The Hong Kong old-old study. The Journals of Gerontology. Series A, Biological Sciences \& Medical Sciences, 52(6), M356-M362. PubMed ID: 9402942 doi:10.1093/gerona/ 52A.6.M356

Huguet, A., Hayden, J.A., Stinson, J., McGrath, P.J., Chambers, C.T., Tougas, M.E., \& Wozney, L. (2013). Judging the quality of evidence in reviews of prognostic factor research: Adapting the GRADE framework. Systematic Reviews, 2(1), 71. PubMed ID: 24007720 doi:10.1186/2046-4053-2-71
Jørgensen, T.S.H., Lund, R., Siersma, V.D., \& Nilsson, C.J. (2017). Interplay between financial assets and social relations on decline in physical function and mortality among older people. European Journal of Ageing, 15(2), 133-142. PubMed ID: 29867298 doi:10. 1007/s10433-017-0437-0

Keskinen, K.E., Rantakokko, M., Suomi, K., Rantanen, T., \& Portegijs, E. (2020). Hilliness and the development of walking difficulties among community-dwelling older people. Journal of Aging and Health, 32(5-6), 278-284. PubMed ID: 30587067 doi:10.1177/0898264 318820448

Koster, A., Penninx, B.W., Bosma, H., Kempen, G.I., Harris, T.B., Newman, A.B., . . . Kritchevsky, S.B. (2005). Is there a biomedical explanation for socioeconomic differences in incident mobility limitation? The Journals of Gerontology. Series A, Biological Sciences \& Medical Sciences, 60(8), 1022-1027. PubMed ID: 16127107 doi:10. 1093/gerona/60.8.1022

LaCroix, A.Z., Guralnik, J.M., Berkman, L.F., Wallace, R.B., \& Satterfield, S. (1993). Maintaining mobility in late life. II. Smoking, alcohol consumption, physical activity, and body mass index. American Journal of Epidemiology, 137(8), 858-869. PubMed ID: 8484377 doi:10.1093/oxfordjournals.aje.a116747

Lang, I.A., Llewellyn, D.J., Langa, K.M., Wallace, R.B., \& Melzer, D. (2008). Neighbourhood deprivation and incident mobility disability in older adults. Age and Ageing, 37(4), 403-410. PubMed ID: 18487260 doi:10.1093/ageing/afn092

Lee, J.S., Kritchevsky, S.B., Tylavsky, F., Harris, T., Simonsick, E.M., Rubin, S.M., \& Newman, A.B. (2005). Weight change, weight change intention, and the incidence of mobility limitation in wellfunctioning community-dwelling older adults. The Journals of Gerontology. Series A, Biological Sciences \& Medical Sciences, 60(8), 1007-1012. PubMed ID: 16127104 doi:10.1093/gerona/60.8.1007

Leveille, S.G., Bean, J., Ngo, L., McMullen, W., \& Guralnik, J.M. (2007). The pathway from musculoskeletal pain to mobility difficulty in older disabled women. Pain, 128(1-2), 69-77. PubMed ID: 17055167 doi:10.1016/j.pain.2006.08.031

Leveille, S.G., Ling, S., Hochberg, M.C., Resnick, H.E., Bandeen-Roche, K.J., Won, A., \& Guralnik, J.M. (2001). Widespread musculoskeletal pain and the progression of disability in older disabled women. Annals of Internal Medicine, 135(12), 1038-1046. PubMed ID: 11747382 doi:10.7326/0003-4819-135-12-200112180-00007

Liljas, A.E.M., Wannamethee, S.G., Whincup, P.H., Papacosta, O., Walters, K., Iliffe, S., . . Ramsay, S.E. (2016). Hearing impairment and incident disability and all-cause mortality in older British community-dwelling men. Age and Ageing, 45(5), 661-666. PubMed ID: 27146303 doi:10.1093/ageing/afw080

Lindberg, O., \& Tilvis, R.S. (1998). Does the metabolic syndrome predict mobility impairment in the elderly? Archives of Gerontology and Geriatrics, 26(2), 131-139. PubMed ID: 18653132 doi:10.1016/ S0167-4943(97)00037-X

Mänty, M., Heinonen, A., Leinonen, R., Törmäkangas, T., Sakari-Rantala, R., Hirvensalo, M., . . Rantanen, T. (2007). Construct and predictive validity of a self-reported measure of preclinical mobility limitation. Archives of Physical Medicine and Rehabilitation, 88(9), 1108-1113. PubMed ID: 17826454 doi:10.1016/j.apmr.2007.06.016

Mänty, M., Heinonen, A., Viljanen, A., Pajala, S., Koskenvuo, M., Kaprio, J., \& Rantanen, T. (2009). Outdoor and indoor falls as predictors of mobility limitation in older women. Age and Ageing, 38(6), 757-761. PubMed ID: 19779051 doi:10.1093/ageing/afp178

Mehta, K.M., Yaffe, K., Brenes, G.A., Newman, A.B., Shorr, R.I., Simonsick, E.M., . . . Covinsky, K.E. (2007). Anxiety symptoms and decline in physical function over 5 years in the health, aging and body composition study. Journal of the American Geriatrics Society, 
55(2), 265-270. PubMed ID: 17302665 doi:10.1111/j.1532-5415. 2007.01041.x

Moher, D., Liberati, A., Tetzlaff, J., \& Altman, D.G. (2009). Preferred reporting items for systematic reviews and meta-analyses: The PRISMA statement. Annals of Internal Medicine, 151(4), 264-269. PubMed ID: 19622511 doi:10.7326/0003-4819-151-4-20090818000135

Moons, K.G., de Groot, J.A., Bouwmeester, W., Vergouwe, Y., Mallett, S., Altman, D.G., . . . Collins, G.S. (2014). Critical appraisal and data extraction for systematic reviews of prediction modelling studies: The CHARMS checklist. PLoS Medicine, 11(10), e1001744. PubMed ID: 25314315 doi:10.1371/journal.pmed.1001744

Moons, K.G.M., Royston, P., Vergouwe, Y., Grobbee, D.E., \& Altman, D.G. (2009). Prognosis and prognostic research: What, why, and how? British Medical Journal, 338, b375. PubMed ID: 19237405 doi:10.1136/bmj.b375

Nilsson, C.J., Avlund, K., \& Lund, R. (2010). Social inequality in onset of mobility disability among older Danes: The mediation effect of social relations. Journal of Aging and Health, 22(4), 522-541. PubMed ID: 20231729 doi:10.1177/0898264309359684

Nilsson, C.J., Lund, R., \& Avlund, K. (2008). Cohabitation status and onset of disability among older Danes: Is social participation a possible mediator? Journal of Aging and Health, 20(2), 235-253. PubMed ID: 18089766 doi:10.1177/0898264307310474

Onder, G., Penninx, B.W., Guralnik, J.M., Jones, H., Fried, L.P., Pahor, M., \& Williamson, J.D. (2003). Sexual satisfaction and risk of disability in older women. The Journal of Clinical Psychiatry, 64(10), 1177-1182. PubMed ID: 14658965 doi:10.4088/JCP. v64n1006

Papachristou, E., Wannamethee, S.G., Lennon, L.T., Papacosta, O., Whincup, P.H., Iliffe, S., \& Ramsay, S.E. (2017). Ability of self-reported frailty components to predict incident disability, falls, and all-cause mortality: Results from a population-based study of older British men. Journal of the American Medical Directors Association, 18(2), 152-157. PubMed ID: 27742583 doi:10.1016/j.jamda.2016.08.020

Pine, Z.M., Gurland, B., \& Chren, M.M. (2000). Report of having slowed down: Evidence for the validity of a new way to inquire about mild disability in elders. The Journals of Gerontology. Series A, Biological Sciences \& Medical Sciences, 55(7), M378-M383. PubMed ID: 10898254 doi:10.1093/gerona/55.7.M378

Pine, Z.M., Gurland, B., \& Chren, M.M. (2002). Use of a cane for ambulation: Marker and mitigator of impairment in older people who report no difficulty walking. Journal of the American Geriatrics Society, 50(2), 263-268. PubMed ID: 12028207 doi:10.1046/j.15325415.2002.50057.x

Polku, H., Mikkola, T.M., Rantakokko, M., Portegijs, E., Törmäkangas, T., Rantanen, T., \& Viljanen, A. (2015). Self-reported hearing difficulties and changes in life-space mobility among communitydwelling older adults: A two-year follow-up study. BMC Geriatrics, 15(1), 121. PubMed ID: 26459630 doi:10.1186/s12877-015-0119-8

Rantakokko, M., Iwarsson, S., Mänty, M., Leinonen, R., \& Rantanen, T. (2012). Perceived barriers in the outdoor environment and development of walking difficulties in older people. Age and Ageing, 41(1), 118-121. PubMed ID: 22086965 doi:10.1093/ageing/afr136

Rantakokko, M., Mänty, M., \& Rantanen, T. (2013). Mobility decline in old age. Exercise and Sport Sciences Reviews, 41(1), 19-25. PubMed ID: 23038241 doi:10.1097/JES.0b013e3182556f1e

Rantakokko, M., Portegijs, E., Viljanen, A., Iwarsson, S., \& Rantanen, T. (2016). Mobility modification alleviates environmental influence on incident mobility difficulty among community-dwelling older people: A two-year follow-up study. PLoS One, 11(4), e0154396. PubMed ID: 27104750 doi:10.1371/journal.pone.0154396
Reynolds, S.L., \& Silverstein, M. (2003). Observing the onset of disability in older adults. Social Science \& Medicine, 57(10), 1875-1889. PubMed ID: 14499512 doi:10.1016/S0277-9536(03)00053-4

Riley, R.D., Moons, K.G.M., Snell, K.I.E., Ensor, J., Hooft, L., Altman, D.G., . . Debray, T.P.A. (2019). A guide to systematic review and meta-analysis of prognostic factor studies. BMJ, 364, k4597. PubMed ID: 30700442 doi:10.1136/bmj.k4597

Sauvaget, C., Tsuji, I., Aonuma, T., \& Hisamichi, S. (1999). Health-life expectancy according to various functional levels. Journal of the American Geriatrics Society, 47(11), 1326-1331. PubMed ID: 10573441 doi:10.1111/j.1532-5415.1999.tb07433.x

Schroll, M., Avlund, K., \& Davidsen, M. (1997). Predictors of five-year functional ability in a longitudinal survey of men and women aged 75 to 80. The 1914-population in Glostrup, Denmark. Aging, 9(1-2), 143-152. PubMed ID: 9177598 doi:10.1007/bf03340140

Simonsick, E.M., Aronson, B., Schrack, J.A., Hicks, G.E., Jerome, G.J., Patel, K.V., . . . Ferrucci, L. (2018). Lumbopelvic pain and threats to walking ability in well-functioning older adults: Findings from the Baltimore Longitudinal Study of Aging. Journal of the American Geriatrics Society, 66(4), 714-720. PubMed ID: 29411349 doi:10. 1111 /jgs. 15280

Simonsick, E.M., Glynn, N.W., Jerome, G.J., Shardell, M., Schrack, J.A., $\&$ Ferrucci, L. (2016). Fatigued, but not frail: Perceived fatigability as a marker of impending decline in mobility-intact older adults. Journal of the American Geriatrics Society, 64(6), 1287-1292. PubMed ID: 27253228 doi:10.1111/jgs. 14138

Simonsick, E.M., Schrack, J.A., Santanasto, A.J., Studenski, S.A., Ferrucci, L., \& Glynn, N.W. (2018). Pittsburgh Fatigability Scale: One-page predictor of mobility decline in mobility-intact older adults. Journal of the American Geriatrics Society, 66(11), 2092-2096. PubMed ID: 30315707 doi:10.1111/jgs.15531

Syddall, H.E., Westbury, L.D., Cooper, C., \& Sayer, A.A. (2015). Selfreported walking speed: A useful marker of physical performance among community-dwelling older people? Journal of the American Medical Directors Association, 16(4), 323-328. PubMed ID: 25523286 doi:10.1016/j.jamda.2014.11.004

Thakral, M., Shi, L., Shmerling, R.H., Bean, J.F., \& Leveille, S.G. (2014). A stiff price to pay: Does joint stiffness predict disability in an older population? Journal of the American Geriatrics Society, 62(10), 1891-1899. PubMed ID: 25333527 doi:10.1111/jgs.13070

Thorpe, R.J., Jr., Koster, A., Kritchevsky, S.B., Newman, A.B., Harris, T., Ayonayon, H.N., . . . Health Aging and Body Composition Study. (2011). Race, socioeconomic resources, and late-life mobility and decline: Findings from the health, aging, and body composition study. The Journals of Gerontology. Series A, Biological Sciences and Medical Sciences, 66(10), 1114-1123. PubMed ID: 21743093 doi:10.1093/gerona/glr102

Tsuji, T., Rantakokko, M., Portegijs, E., Viljanen, A., \& Rantanen, T. (2018). The effect of body mass index, lower extremity performance, and use of a private car on incident life-space restriction: A two-year follow-up study. BMC Geriatrics, 18(1), 271. PubMed ID: 30409120 doi:10.1186/s12877-018-0956-3

van den Berg, T., Heymans, M.W., Leone, S.S., Vergouw, D., Hayden, J.A., Verhagen, A.P., \& de Vet, H.C.W. (2013). Overview of datasynthesis in systematic reviews of studies on outcome prediction models. BMC Medical Research Methodology, 13(1), 42. PubMed ID: 23497181 doi:10.1186/1471-2288-13-42

van den Brink, C.L., Tijhuis, M., van den Bos, G.A., Giampaoli, S., Kivinen, P., Nissinen, A., \& Kromhout, D. (2004). Effect of widowhood on disability onset in elderly men from three European countries. Journal of the American Geriatrics Society, 52(3), 353-358. PubMed ID: 14962148 doi:10.1111/j.1532-5415.2004.52105.x 
Verbrugge, L.M., \& Jette, A.M. (1994). The disablement process. Social Science \& Medicine, 38(1), 1-14. PubMed ID: 8146699 doi:10.1016/ 0277-9536(94)90294-1

Viljanen, A., Kulmala, J., Rantakokko, M., Koskenvuo, M., Kaprio, J., \& Rantanen, T. (2012). Fear of falling and coexisting sensory difficulties as predictors of mobility decline in older women. The Journals of Gerontology. Series A, Biological Sciences \& Medical Sciences, 67(11), 1230-1237. PubMed ID: 22546957 doi:10.1093/gerona/gls134

Visser, M., Simonsick, E.M., Colbert, L.H., Brach, J., Rubin, S.M., Kritchevsky, S.B., . . Harris, T.B. (2005). Type and intensity of activity and risk of mobility limitation: The mediating role of muscle parameters. Journal of the American Geriatrics Society, 53(5), 762-770. PubMed ID: 15877550 doi:10.1111/j.1532-5415.2005. 53257.x

Webber, S.C., Porter, M.M., \& Menec, V.H. (2010). Mobility in older adults: A comprehensive framework. Gerontologist, 50(4), 443-450. PubMed ID: 20145017 doi:10.1093/geront/gnq013
Weiss, C.O., Wolff, J.L., Egleston, B., Seplaki, C.L., \& Fried, L.P. (2012). Incident preclinical mobility disability (PCMD) increases future risk of new difficulty walking and reduction in walking activity. Archives of Gerontology and Geriatrics, 54(3), e329-e333. PubMed ID: 21944428 doi:10.1016/j.archger.2011.08.018

Wolff, R.F., Moons, K.G.M., Riley, R.D., Whiting, P.F., Westwood, M., Collins, G.S., . . PROBAST Group. (2019). PROBAST: A tool to assess the risk of bias and applicability of prediction model studies. Annals of Internal Medicine, 170(1), 51-58. PubMed ID: 30596875 doi:10.7326/M18-1376

World Bank. (2020). World Bank list of economies. Retrieved from https:// datahelpdesk.worldbank.org/knowledgebase/articles/906519-worldbank-country-and-lending-groups

Yeom, H.A., Fleury, J., \& Keller, C. (2008). Risk factors for mobility limitation in community-dwelling older adults: A social ecological perspective. Geriatric Nursing, 29(2), 133-140. PubMed ID: 18394514 doi:10.1016/j.gerinurse.2007.07.002 